\title{
Here I am: The cortical correlates of visual self-recognition
}

\author{
Devue Christel $^{\mathrm{a}}$, Collette Fabienne ${ }^{\mathrm{a}, \mathrm{b}}$, Balteau Evelyne ${ }^{\mathrm{b}}$, Degueldre Christian ${ }^{\mathrm{b}}$, Luxen Andréb, Maquet Pierre ${ }^{\mathrm{b}}$, \\ Brédart Serge ${ }^{\mathrm{a}}$ \\ ${ }^{a}$ Department of Cognitive Science, University of Liège, 5, Boulevard du Rectorat (Bat. B32), 4000 Liège, Belgium ${ }^{b}$ Cyclotron Research \\ Centre, University of Liège, Liège, Belgium
}

\begin{abstract}
Recently, interest in the neural correlates of self-recognition has grown. Most studies concentrate on self-face recognition. However, there is a lack of convergence as to precise neuroanatomical locations underlying self-face recognition. In addition, recognition of familiar persons from bodies has been relatively neglected. In the present study, cerebral activity while participants performed a task in which they had to indicate the real appearance of themselves and of a gender-matched close colleague among intact and altered pictures of faces and bodies was measured. The right frontal cortex and the insula were found to be the main regions specifically implicated in visual self-recognition compared with visual processing of other highly familiar persons. Moreover, the right anterior insula along with the right anterior cingulate seemed to play a role in the integration of information about oneself independently of the stimulus domain. The processing of self-related pictures was also compared to scrambled versions of these pictures. Results showed that different areas of the occipito-temporal cortex were more or less recruited depending on whether a face or a body was perceived, as it has already been reported by several recent studies. The implication of present findings for a general framework of person identification is discussed.
\end{abstract}

Keywords : Body shape recognition ; face recognition ; familiar face ; functional magnetic resonance imagery ; self-processing

\section{INTRODUCTION}

The face is a physical characteristic that is critical to the identification of people we meet. The recognition of one's own face is also important. The ability to recognize one's own face appears to participate in maintaining a sense of self (Platek et al., 2004b). To recognize oneself, one must have the ability to build and retrieve a representation of one's physical appearance, and to regard the self as a different entity from others. Hence, many researchers view self-recognition as an indicator of self-awareness (see Gallup et al., 2003). Recently, the question of whether there are neural mechanisms which are distinctively related to the process of self-recognition (as compared with the recognition of other familiar people) has drawn the attention of a growing number of cognitive neuroscientists (for recent reviews see Gillihan and Farah, 2005; Keenan et al., 2003a, b).

The examination of split-brain patients has demonstrated that both hemispheres are capable of self-recognition (Sperry et al., 1979; Uddin et al., 2005b). However, evidence that self-recognition preferentially involves the right hemisphere has been reported. Several studies have indicated a left-hand advantage in self-face recognition tasks in healthy participants (Keenan et al., 1999; Keenan et al., 2000a, b; Platek and Gallup, 2002; Platek et al., 2004b; Zhu et al, 2004). Because of contralateral motor control, this left-hand advantage supports the view that the right hemisphere is predominant in self-recognition. A right hemispheric advantage for self-face recognition in a callosotomy patient has also been reported (Keenan et al., 2003c; but see Turk et al., 2002, for a left hemisphere advantage in another split-brain patient). In addition, patients who were undergoing Wada tests were shown images of themselves morphed with a famous face during right and left hemispheric anesthetization.

After the anesthesia has subsided, patients were asked about the face they were shown. They were more likely to report having seen themselves after the anesthetization of the left hemisphere than after the anesthetization of the right hemisphere (Keenan et al., 2001). Finally, healthy participants showed greater right hemispheric activity, as measured by evoked potentials induced by transcranial magnetic stimulation, while presented with morphed or masked pictures of their own face as opposed to pictures of another person (Keenan et al., 2001; Théoret et al., 2004).

However, there are studies that support the opposite view that the left hemisphere has a dominant role in selfrecognition. In one study, already mentioned here above, a split-brain patient was presented with morphed images blending his own face with a familiar person's face (Turk et al., 2002). These images were presented separately to the left and to the right hemispheres. In one condition the patient's task was to determine whether a presented image was himself while in another condition his task was to determine whether the image was the familiar person. The rate of self-detection was higher when the images were presented to the left than to the right hemisphere. On the opposite, detection of the familiar person was better when the images were presented to the right than to the left hemisphere. More recently, healthy participants were asked to choose which of two chimeric faces (one made from the left half and one made from the right half of their face) looked more like themselves 
(Brady et al., 2004). They showed a bias for the composite made from the half face that lies in their right visual field when they look at themselves in the mirror. When asked to make the same choice for similar images of a friend, they showed the opposite bias, i.e., they preferentially chose the composite made from the half face that lies in their left visual field when they look at their friend. Such results suggest that the left hemisphere is dominant for self-recognition and the right hemisphere is dominant for the recognition of others.

Results from functional neuroimaging studies of self-recognition using functional magnetic resonance imaging (fMRI) and PET are also controversial. Some studies concluded that the right prefrontal regions are critical for self-face recognition (Platek et al., 2004a; Keenan et al., 2003b). More recently, Uddin et al. (2005a) reported that a neural network in the right hemisphere including the inferior frontal gyrus and the inferior parietal lobule is activated by the recognition of the self-face. In another experiment, Uddin et al. (2006) also confirmed the implication of the right inferior parietal lobule during self-face recognition and demonstrated for the first time the existence of a causal relationship between this region and self-recognition. To do so, they used a lowfrequency repetitive transcranial magnetic stimulation to induce a virtual lesion in this region. This manipulation decreased the sensitivity of participants to detect their own face among morphed images of themselves and another highly familiar person while this manipulation had no effect when it was applied on the same region on the left side. However, there is also evidence suggesting bilateral involvement in self-face recognition. Kircher et al. $(2000,2001)$ reported activation in the right limbic system, left prefrontal cortex and temporal cortex during self-face processing. In a PET study, Sugiura et al. (2000) found an implication of a large bilateral network involving the bilateral prefrontal cortex, the fusiform gyrus, the insula and the putamen on the left side, the supramarginal gyrus, the anterior cingulate, the presupplementary motor area and the hypothalamus on the right side during self-face recognition. More recently, Sugiura et al. (2005) conducted an fMRI study aimed at identifying the cortical mechanisms of self-face recognition by controlling the selectivity of the activation for the self-face. To do so, they compared the activity elicited for the self-face to that found with faces of different degrees of familiarity (i.e., a friend, an experimenter and a prelearned unfamiliar face) and excluded activation that could be explained by these differences of familiarity. They observed selective activation of the right occipito-temporo-parietal junction and frontal operculum, as well as in the left fusiform gyrus during self-face recognition. Platek et al. (2006) contrasted cerebral responses to self-face and another personally familiar face and also found an implication of both hemispheres (superior frontal gyrus, medial frontal and inferior parietal lobes on the right, and middle temporal gyrus on the left) during self-face identification.

Our first aim was to examine the cortical mechanisms of visual self-face recognition. The lack of convergence as to precise anatomical locations underlying self-face recognition motivated the present study. Moreover, previous studies considerably differed between each other with regard to the familiarity of the control face compared with the self-face. Depending on the studies and on the contrasts formally used in these studies, the control face was unfamiliar (Sugiura et al., 2000), recently learned (Sugiura et al., 2005), famous (Platek et al., 2004a) or personally known to the participant (Kircher et al., 2000, 2001; Platek et al., 2006; Sugiura et al., 2005; Uddin et al., 2005a). Since control of familiarity is more efficient when the self-face is compared with a highly familiar face, we used a personally known, gender-matched, person as the control face in the current study. Because distinguishing two highly familiar persons from each other is presumably very easy, we designed a task in which participants would have to identify their real facial appearance and that of their colleague among intact and altered pictures presented during a first event-related scanning session. In addition, the use of altered pictures allowed to increase stimulus variability and helped to decrease repetition suppression of the BOLD signal (GrillSpector et al., 2006). However, we were mainly interested in the processing of intact stimuli because responses to altered views of these faces might not reflect usual processing of such familiar stimuli (Platek et al., 2004a, b). In other words, participants' task consisted in an "intact-altered" judgment both on pictures of themselves or of a close colleague, all these pictures being presented at random. The facial alterations consisted in moving the eyes inwards or outwards. To increase the statistical power of our analysis, we used a priori regions of interest found to be elicited in studies using similar contrasts (i.e., self-face minus other familiar face). These regions were the right inferior frontal gyrus (Platek et al., 2004a; Uddin et al., 2005a), the left inferior frontal gyrus (Kircher et al., 2000), the right superior frontal gyrus (Platek et al., 2006), the right middle frontal gyrus (Platek et al., 2006), the right medial frontal gyrus (Platek et al., 2006), the left middle temporal gyrus (Platek et al., 2006), the left fusiform gyrus (Kircher et al., 2001) and the right inferior parietal lobule (Platek et al., 2006; Uddin et al., 2005a).

In addition, Gillihan and Farah (2005) recently noticed that there is a lack of studies of self-recognition from the body shape or body parts. Hence, the second aim of the current study was to investigate the cortical correlates of visual self-body recognition by assessing whether specific cortical regions underlie the own body recognition compared with the recognition of another familiar person's body. In a second event-related scanning session, we asked participants to identify their real body-shape appearance and that of their colleague among intact and altered pictures. The body alterations consisted in increasing or decreasing the waist-to-hip ratio by changing the width of the hips. Again, the alterations were introduced to increase the difficulty of the task and to induce some 
variability in the stimuli. Due to the explorative nature of this comparison and to the lack of studies investigating this specific topic, we tentatively reported regions activated when comparisons between the processing of the self-body and the processing of another highly familiar person's body were examined.

Importantly, the use of body shapes as stimuli was also motivated by the third aim of determining which cerebral regions are selectively activated by self-processing regardless of the domain of presented stimulus (body or face). In some previous studies, the neural correlates of self-processing were explored using auditory presentations of the own name (e. g., Holeckova et al., 2006; Perrin et al., 1999, 2005). However, contrary to the self-face or body, the own name is not an exclusively self-referential stimulus since it can be shared with other people. The self-voice is another type of auditory self-referential stimulus. It has the advantage of not being shared with other people. However, the use of such a stimulus may also be problematic. Indeed, hearing our own voice played back does not account for bone conductance and therefore a recording of our voice rarely sounds like our own voice heard from inside. Moreover, manipulating voices and faces is hardly comparable ${ }^{1}$. Hence, in addition to the self-face, the self-body was used instead of the own name or voice. Thus, for that purpose, data related to the self-face and data related to the self-body were collapsed and compared with the data related to the processing of the colleague's face and body. To increase the statistical power of our analysis on this contrast, we used a priori regions of interest found to be activated in studies investigating different tasks associated with autoreferential processing such as judgment of adjectives/sentences as self-descriptive or judgment of actions as self generated. These regions were the right/medial prefrontal cortex (Fossati et al., 2003; Kelley et al., 2002), the right anterior cingulate gyrus (Craik et al., 1999), the anterior insula (Farrer and Frith, 2002), the bilateral precuneus (Kircher et al., 2000, 2002; Ruby and Decety, 2001), the left inferior frontal gyrus (Craik et al., 1999; Kircher et al., 2002) and the left superior frontal gyrus (Ruby and Decety, 2001; Seger et al., 2004).

To summarize, in order to answer our three main questions, we used an event-related paradigm in which we examined cerebral activity elicited by the presentation of pictures depicting the face and the body of the participant and those of a close colleague. The participants' task was to discriminate between intact and altered pictures of themselves and of another highly familiar person. The alterations were used to prevent a fast habituation by inducing some variability among the stimuli and also to increase the difficulty of the task, the main interest being the participant's ability to correctly identify intact bodies or faces. The intact self minus intact colleague contrasts presented below thus reflected the BOLD signal changes found when participants processed their real physical appearance compared to that of another personally familiar individual.

Finally, as it has been suggested by several recent previous studies (Downing et al., 2001, 2006; Peelen and Downing, 2005; Schwarzlose et al., 2005; see also Glila and Dehaene-Lambertz, 2005 for an ERP study) that faces and bodies are associated with distinct neural correlates, we included two event-related control sessions in which participants passively viewed intact and scrambled pictures of their face (within one of these control sessions) or of their body (within the other control session). These two sessions would allow to determine the cerebral areas associated respectively with general face and body shape processing and would also allow to compare the neural substrates associated with the processing of these two kinds of stimuli. We used a priori regions of interest found to be elicited in studies comparing object or face processing to other kinds of stimuli (i.e., tools, letters or textures, scrambled pictures) processing. Regarding face recognition, these regions were the bilateral fusiform gyrus (Peelen and Downing, 2005, Puce et al., 1996), the right ventral occipital cortex (Peelen and Downing, 2005), the right occipito-temporal/ inferior occipital sulci (Puce et al., 1996) and the bilateral lateral neocortex (Puce et al., 1996). Regarding body recognition, these regions were the right fusiform gyrus (Peelen and Downing, 2005) and the bilateral inferior temporal sulcus (Peelen and Downing, 2005).

\section{RESULTS}

\section{1. Behavioral data}

The data from one participant whose response accuracy were more than $2.5 \mathrm{SD}$ under the mean of the sample were discarded.

\section{1. 1. Accuracy}

A 2 (Stimulus domain: face/body) x 2 (Identity: self/colleague) x 2 (Stimulus appearance: intact/altered) repeated measures ANOVA was carried out on proportions of correct responses and did not reveal any significant main effect, all $F \mathrm{~s}<2, p>0.05$. In addition, no interaction was significant except the Stimulus domain $\mathrm{x}$ Stimulus appearance interaction, $F(1,18)=13.16, p<0.01$. HSD Tukey post hoc comparisons indicated that intact faces were better recognized than intact bodies $(p<0.05)$ and that altered bodies were better recognized than intact bodies $(p<0.05)$. No other difference reached significance. Descriptive data are presented in Table 1.

\footnotetext{
${ }^{1}$ We thank an anonymous reviewer for this suggestion.
} 


\section{1. 2. Reaction times}

Reaction times below $300 \mathrm{~ms}$ were excluded from the analyses. A 2 (Stimulus domain: face/body) x 2 (Identity: self/colleague) x 2 (Stimulus appearance: intact/altered) repeated measures ANOVA was performed on median correct reaction times and showed a main effect of Stimulus domain, $F(1,18)=14.37, p<0.01$, participants being faster for faces $(M=840, S D=240)$ than for bodies $(M=949, S D=279)$, and a main effect of Identity, $F(1,18)$ $=9.84, p<0.01$, participants being faster for themselves $(\mathrm{M}=879, \mathrm{SD}=230)$ than for their colleague $(\mathrm{M}=910$, $\mathrm{SD}=234)$. There was also a main effect of Stimulus appearance, $F(1,18)=10.53, p<0.01$, intact stimuli $(\mathrm{M}=$ $923, \mathrm{SD}=267)$ being recognized more slowly than altered ones $(\mathrm{M}=866, \mathrm{SD}=211)$. No interaction was significant (all $p s>0.05$ ) except the Stimulus domain $x$ Stimulus appearance interaction, $F(1,18)=14.67, p<0.01$. Tukey post hoc comparisons indicated that intact faces were identified faster than intact bodies $(p<0.001)$, altered faces were identified faster than altered bodies $(p<0.001)$, altered bodies were identified faster than intact bodies $(p<0.001)$ and finally intact faces were identified faster than altered bodies $(p<0.01)$. No other comparison reached significance (see Table 1 for descriptive data).

These results showed that processing faces was faster than processing bodies, and that processing self-relevant stimuli was faster than processing stimuli depicting a colleague. However, Identity and Stimulus domain did not significantly impact the proportions of accurate responses. The effect of Stimulus appearance was not of first importance here since we were interested in the analysis of BOLD signals elicited by intact pictures.

Table 1 - Mean median reaction times in milliseconds and mean proportion of correct responses (in italics) as a function of the Identity and of the Stimulus domain (standard deviations are between parentheses)

\begin{tabular}{lllll}
\hline Identity & \multicolumn{4}{c}{ Stimulus domain } \\
\cline { 2 - 5 } & \multicolumn{2}{c}{ Face } & \multicolumn{2}{c}{ Body } \\
\cline { 2 - 5 } & \multicolumn{1}{c}{ Self } & \multicolumn{1}{c}{ Colleague } & \multicolumn{1}{c}{ Self } & \multicolumn{1}{c}{ Colleague } \\
\hline Stimulus & appearance & & & \\
Intact & $847(152)$ & $859(137)$ & $955(167)$ & $1031(169)$ \\
& $0.96(0.04)$ & $0.94(0.06)$ & $0.91(0.08)$ & $0.89(0.09)$ \\
Altered & $816(90)$ & $838(128)$ & $897(127)$ & $911(149)$ \\
& $0.93(0.08)$ & $0.92(0.08)$ & $0.95(0.04)$ & $0.95(0.06)$ \\
\hline
\end{tabular}

The performance on altered items is given for information.

\section{2. Imaging data}

First, intact faces and bodies were compared with, respectively, scrambled faces and bodies in order to check whether the same cerebral areas associated respectively with face and body processing as those reported earlier (Downing et al., 2001, 2006; Peelen and Downing, 2005; Schwarzlose et al., 2005) were activated. Faces and bodies elicited responses in close but segregated regions of the occipital cortex (Fig. 1). Perception of faces was associated with a bilateral cerebral activity in the middle occipital gyrus, extending to the fusiform gyrus on the right and to the cerebellum on the left, as well as with activation of a large frontal area on the right side (middle and inferior frontal gyrus, and medial/superior frontal gyrus) (see Table 2). Perception of bodies was associated with activity in the fusiform and lateral occipital complex bilaterally, and with activity in the left middle occipital gyrus (see Table 2). These findings are consistent with previous literature (Downing et al., 2001, 2006; Peelen and Downing, 2005; Schwarzlose et al., 2005). Direct comparisons between faces and bodies are reported in Table 2 (contrasts (3) and (4)).

After having identified the regions implicated in the processing of faces and bodies, we examined the contrasts of central interest in this study, i.e., the contrasts that assessed which cortical areas are specifically implicated in the processing of self related pictures and in the processing of pictures depicting another highly familiar person.

\section{2. 1. Intact own fac >intact colleague's face}

A significant response was identified in the right inferior frontal gyrus (Fig. 2), consistent with literature (Keenan et al., 2000c; Kircher et al., 2000, 2001; Platek et al., 2004a, 2006; Sugiura et al., 2000; Uddin et al., 2005a), as well as in the right insula. Contrary to previous studies (Kircher et al., 2000, 2001; Sugiura et al., 2000, 2005), no significant implication of the left fusiform was found.

\section{2. 2. Intact colleague's face >intact own face}

The processing of the colleague's face elicited activity in the right superior temporal gyrus. With a priori locations of interest defined from studies that reported a contrast as close as possible as the present one (other highly familiar face minus own face, Platek et al., 2006; Uddin et al., 2005a), a significant differential response 
was observed in the left precuneus and in the left superior temporal gyrus. However, parameter estimates showed that these areas were actually differently deactivated for the colleague's face and for the self-face (see Fig. 3 for an example).

Fig. 1 - Illustration of brain activity observed when intact pictures (faces and bodies) were compared to scrambled pictures. Regions that showed activity associated with the processing of faces are boxed in black; regions that showed activity associated with the processing of bodies are circled in white. (A) Regions with significant $r C B F$ increase (corrected p value <0.05) are superimposed upon a Tl-weighted magnetic resonance imaging slice normalized into the MNI space. Coordinates of all significant regions are given in Table 2. Coronal sections are shown respectively 80 and $55 \mathrm{~mm}$ posterior to the anterior commissure. (B) Mean parameter estimates (arbitrary units) in the regions detected in the contrast Face-Scrambled are displayed for, from left to right, Intact own face, Scrambled own face, Intact own body and Scrambled own body. (C) Mean parameter estimates (arbitrary units) in the regions detected in the contrast Body-Scrambled are displayed for, from left to right, Intact own face, Scrambled own face, Intact own body and Scrambled own body. Error bars represent SEM.

\section{A. \\ Middle occipital cortex}
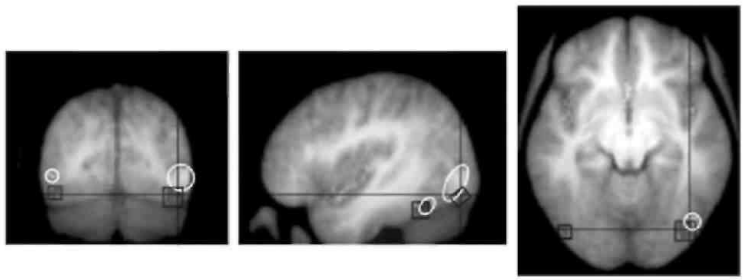

\section{Fusiform gyrus}

B.
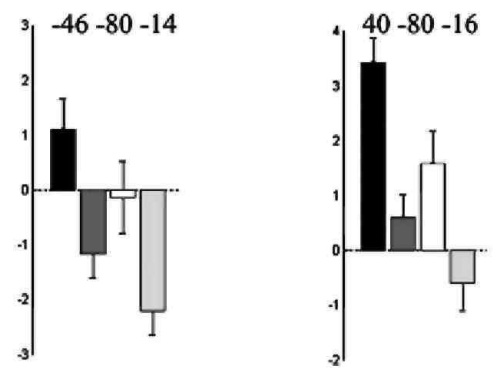

c.
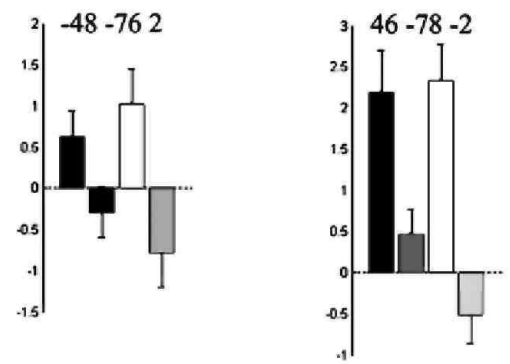

Left
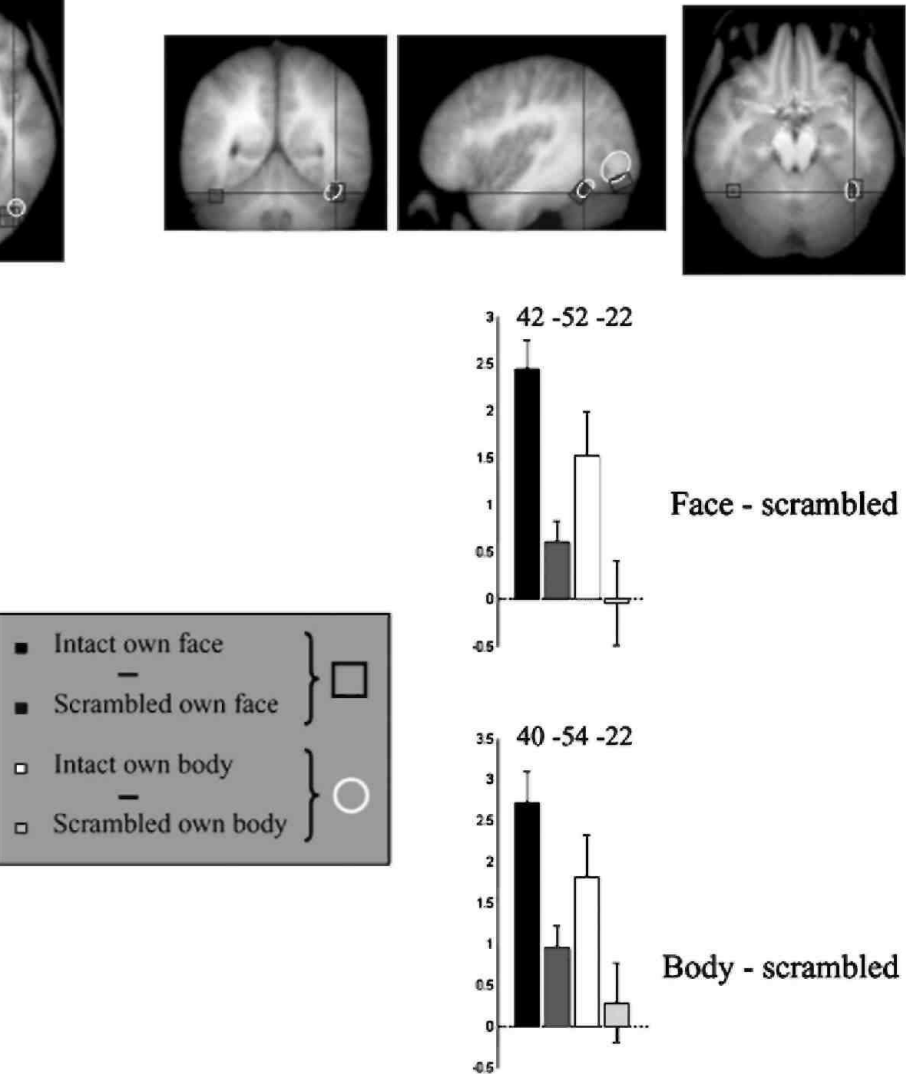

Right 
Fig. 2 - Illustration of brain activation elicited in the right inferior frontal gyrus by the recognition of the intact own face in comparison with the recognition of another highly familiar face. Coordinates of all significant regions are given in Table 2. (Left) Region with significant rCBF increase (corrected at $p<0.05$ after applying small volume corrections) is superimposed upon a Tl-weighted magnetic resonance imaging slice normalized into MNI. Coronal section is shown $32 \mathrm{~mm}$ anterior to the anterior commissure. (Right) Mean parameter estimates (arbitrary units) in the right inferior frontal gyrus for Intact own face and Intact colleague's face. Error bars represent SEM.
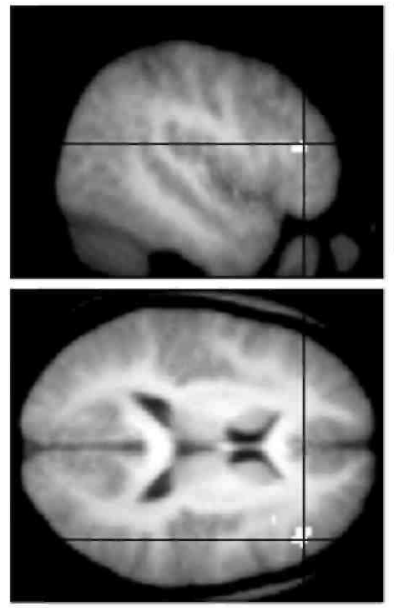
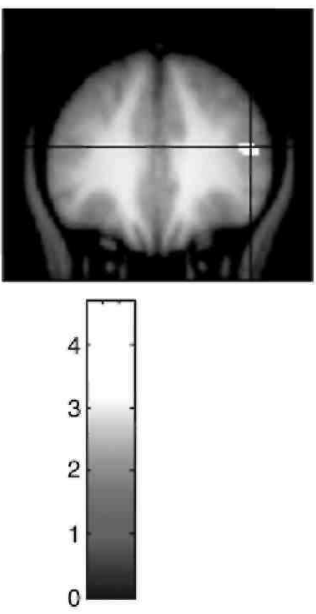

R inferior frontal gyrus 483214

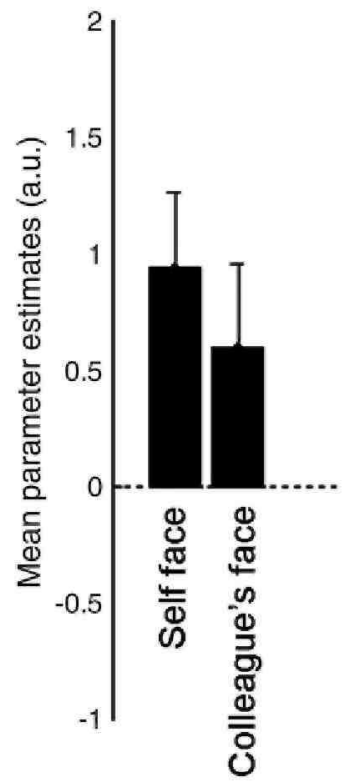

Fig. 3 - Illustration of brain deactivation in the right superior temporal gyrus observed with the comparison of a familiar face to the own face. Coordinates of the region are given in Table 2. (Left) Regions with significant $r C B F$ decrease (corrected at $p<0.05$ ) are superimposed upon a Tl-weighted magnetic resonance imaging slice normalized into MNI. Coronal section is shown $28 \mathrm{~mm}$ posterior to the anterior commissure. (Right) Mean parameter estimates (arbitrary units) in the right insula for Intact own face and Intact colleague's face. Error bars represent SEM.
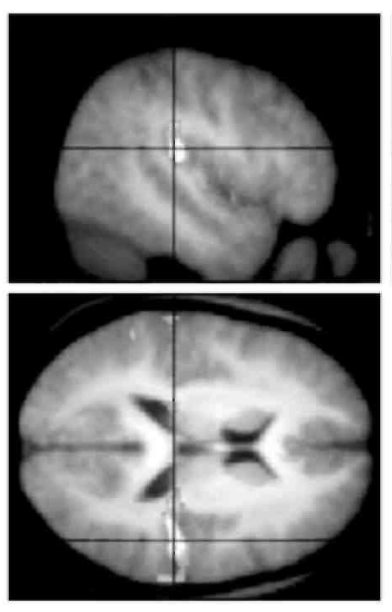
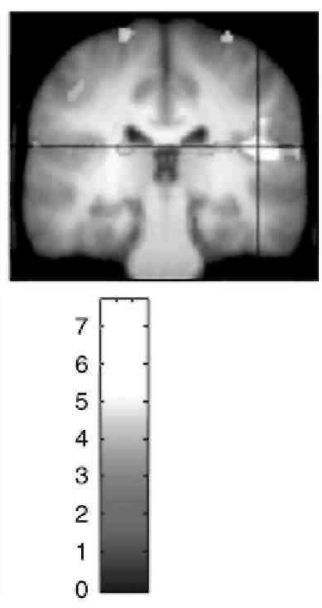

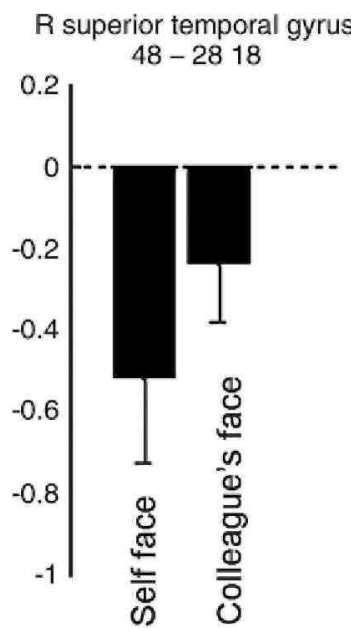

\section{2. 3. Intact own body>intact colleague's body}

Significant responses were detected at a threshold of $p_{\text {uncorrected }}=0.001$ in the right superior frontal sulcus, right cingulate cortex, left inferior frontal gyrus, as well as in the anterior insula bilaterally. None of these results survived correction for multiple comparisons. 


\section{2. 4. Intact colleague's body>intact own body}

Significant responses were detected at a threshold of $p_{\text {uncorrected }}=0.001$ in the left parietal opercule and in a right medial temporal structure close of the lateral part of the hippocampus. None of these results survived correction for multiple comparisons.

Finally, we examined whether specific regions were implicated in the processing of self-related pictures independently of the Stimulus domain.

\section{2. 5. Intact self $>$ intact colleague}

This analysis was based on a priori locations from previous literature related to self-processing. Interestingly, when activation associated with seeing intact stimuli depicting the colleague (faces and bodies) was substracted from activation due to seeing stimuli depicting the participant herself, a significant response was found in the right anterior insula, consistent with literature (Farrer and Frith, 2002; Fink et al., 1996; Kircher et al., 2000, 2001). The response in the right dorsal anterior cingulate also tended to be significant $(Z=3.78, p=0.058)$, consistent with previous findings (Craik et al., 1999; for a review, see Northoff and Bermpohl, 2004) (see Fig. 4).

Fig. 4 - Illustration of brain (de)activation in the right inferior frontal gyrus (top) and right anterior cingulate (bottom) observed for the processing of oneself by comparison to the processing of another person regardless of the Stimulus domain. Coordinates of the regions are given in Table 2. (Left) Regions with significant rCBF increase/decrease (corrected at $p<0.05$ after applying small volume corrections) are superimposed upon a Tlweighted magnetic resonance imaging slice normalized into MNI. Coronal section is shown 32 mm anterior to the anterior commissure. (Right) Mean parameter estimates (arbitrary units) in the right inferior frontal gyrus and in the right anterior cingulate for, from left to right, Intact own face, Intact colleague's face, Intact own body and Intact colleague's body. Error bars represent SEM.
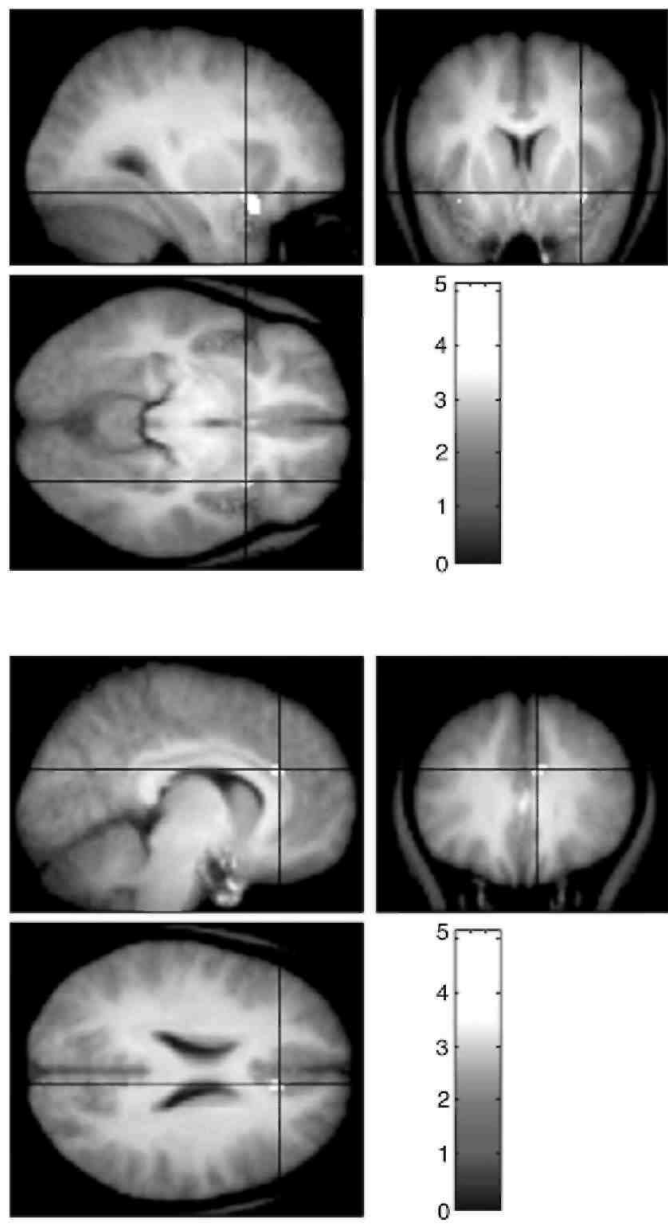

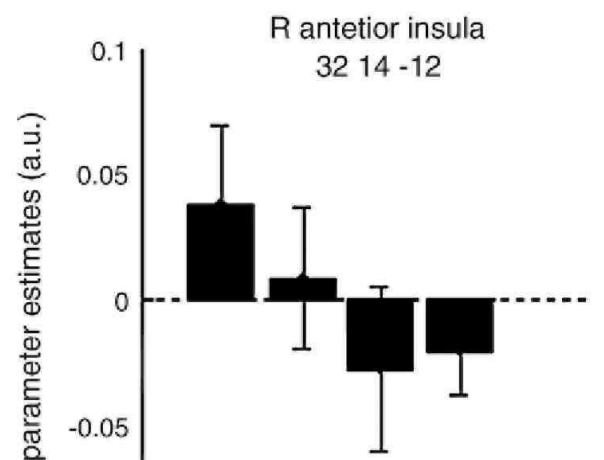

产

$\sum_{\mathbb{\Phi}}^{\mathbb{\Phi}}-0.1$

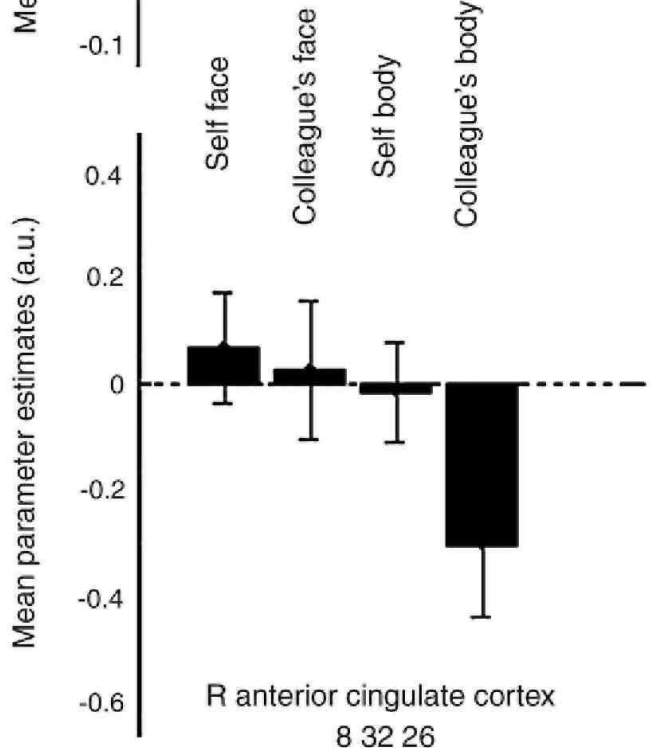


Table 2 - Significant BOLD signal changes in relevant contrasts

\begin{tabular}{|c|c|c|c|c|c|c|}
\hline Anatomical region & Hemisphere & No. voxels & $x$ & $y$ & $z$ & $\begin{array}{c}\text { SPM }\{Z\}- \\
\text { value }\end{array}$ \\
\hline \multicolumn{7}{|l|}{ 1) Intact own face > Scrambled } \\
\hline \multirow[t]{2}{*}{ Cerebellum/middle occipital gyrus } & $\mathrm{L}$ & 1355 & -42 & -54 & -26 & $5.48 *$ \\
\hline & & & -46 & -80 & -14 & $5.28 *$ \\
\hline \multirow[t]{2}{*}{ Middle occipital gyrus/fusiform gyrus } & $\mathrm{R}$ & 2282 & 40 & -80 & -16 & $5.90 *$ \\
\hline & & & 42 & -52 & -22 & $5.69 *$ \\
\hline \multirow[t]{2}{*}{ Inferior frontal gyrus } & $\mathrm{R}$ & 220 & 42 & 34 & 6 & 4. $07 *$ \\
\hline & & & 44 & 24 & 0 & 3. $23 *$ \\
\hline \multirow[t]{2}{*}{ Middle frontal gyrus } & $\mathrm{R}$ & 629 & 46 & 8 & 44 & 4. $49 *$ \\
\hline & & & 40 & -2 & 52 & 3. $77 *$ \\
\hline \multirow[t]{2}{*}{ Medial/superior frontal gyrus } & $\mathrm{R}$ & 313 & 10 & 16 & 50 & 4. $13^{*}$ \\
\hline & & & 6 & 6 & 62 & 4. $03 *$ \\
\hline \multicolumn{7}{|l|}{ 2) Intact own body > Scrambled } \\
\hline \multirow{3}{*}{$\begin{array}{l}\text { Lateral occipital complex/middle occipital } \\
\text { gyrus/fusiform gyrus }\end{array}$} & $\mathrm{L}$ & 2304 & -40 & -72 & -6 & $5.21 *$ \\
\hline & & & -48 & -76 & 2 & $5.06^{*}$ \\
\hline & & & -44 & -68 & -20 & 4. $85^{*}$ \\
\hline Lateral occipital complex & $\mathrm{R}$ & 3087 & 46 & -78 & -2 & $6.15^{*}$ \\
\hline Fusiform gyrus & & & 40 & -54 & -22 & $5.69 *$ \\
\hline \multicolumn{7}{|l|}{ 3) Intact faces > Intact bodies } \\
\hline Medial frontal gyrus & $\mathrm{R}$ & 28 & 8 & 26 & -16 & 4. 74 \\
\hline \multicolumn{7}{|l|}{ 4) Intact bodies > Intact faces } \\
\hline \multirow[t]{2}{*}{ Inferior temporal gyrus/middle temporal gyrus } & $\mathrm{R}$ & 1077 & 52 & -70 & -2 & 4. $91 *$ \\
\hline & & & 42 & -76 & 10 & 4. $50 *$ \\
\hline Middle temporal gyrus & $\mathrm{L}$ & 593 & -42 & -80 & 16 & 4. $17 *$ \\
\hline Temporal lobe/fusiform gyrus & $\mathrm{R}$ & 57 & 34 & -54 & -10 & 3. $83 \#$ \\
\hline Fusiform gyrus & $\mathrm{R}$ & 14 & 34 & -52 & -12 & 3. $67 \#$ \\
\hline \multicolumn{7}{|l|}{ 5) Intact own face > Intact colleague's face } \\
\hline Insula & $\mathrm{R}$ & 2 & 38 & 22 & 16 & 3. $21 \#$ \\
\hline Inferior frontal gyrus & $\mathrm{R}$ & 5 & 48 & 32 & 14 & 3. $20 \#$ \\
\hline \multicolumn{7}{|l|}{ 6) Intact colleague's face > Intact own face } \\
\hline \multirow[t]{3}{*}{ Superior temporal gyrus } & $\mathrm{R}$ & 730 & 48 & -28 & 18 & $5.06^{*}$ \\
\hline & & & 40 & -30 & 18 & 4. $29 *$ \\
\hline & & & 68 & -22 & 12 & $3.96^{*}$ \\
\hline Superior temporal gyrus & $\mathrm{L}$ & 39 & -56 & -48 & 16 & 4. $04 \#$ \\
\hline Precuneus & $\mathrm{L}$ & 61 & -6 & -42 & 56 & 4. $35 \#$ \\
\hline \multicolumn{7}{|l|}{ 7) Intact own body > Intact colleague's body } \\
\hline Superior frontal sulcus & $\mathrm{R}$ & 41 & 26 & 52 & 2 & 4. $01 \sim$ \\
\hline Anterior insula & $\mathrm{R}$ & 82 & 32 & 18 & -16 & 3. 81 \\
\hline Cingulate gyrus & $\mathrm{R}$ & 32 & 12 & 26 & 32 & 3. 69 \\
\hline Inferior frontal gyrus & $\mathrm{L}$ & 39 & -36 & 36 & 16 & 3. 57 \\
\hline Anterior insula & $\mathrm{L}$ & 27 & -36 & 12 & -14 & 3. $43 \sim$ \\
\hline \multicolumn{7}{|l|}{ 8) Intact colleague's body > Intact own body } \\
\hline \multirow[t]{2}{*}{ Parietal opercule } & $\mathrm{L}$ & 43 & -48 & -10 & 18 & 4. $10 \sim$ \\
\hline & & 48 & -22 & 10 & 24 & 3. 88 \\
\hline Medial temporal structure & $\mathrm{R}$ & 16 & 40 & -10 & -30 & $3.94 \sim$ \\
\hline \multicolumn{7}{|l|}{ 9) Intact self > Intact colleague } \\
\hline Anterior cingulate cortex & $\mathrm{R}$ & 15 & 8 & 32 & 26 & 3. $78 \#$ \\
\hline Anterior insula & $\mathrm{R}$ & 10 & 32 & 14 & -12 & 3. $98 \#$ \\
\hline
\end{tabular}

Anterior insula

$\begin{array}{llll}10 & 32 & 14 & -12 \\ \text { isons over the whole brain volume }\left(^{*}\right) \text { or }\end{array}$ small volumes of interest (\#). , Significant at $p<0.001$, uncorrected. 


\section{DISCUSSION}

The present study had three main objectives. First, it was aimed at clarifying the specific neural correlates associated with the recognition of the self-face compared with the recognition of another highly familiar and personally known person. Second, we wanted to test whether specific neural substrates are implicated in the recognition of the self-body in comparison with the recognition of another highly familiar body. Finally, it was tested whether there are specific regions implicated in self-processing independently of the stimulus domain. In order to answer these three questions, we measured BOLD responses elicited while the participants' task was to discriminate between intact and altered pictures of themselves and of a close colleague. An additional objective of that study was to verify, from our two control sessions, whether the areas of the cortex activated by faces and bodies were similar to those that have been recently reported (Downing et al., 2001, 2006; Peelen and Downing, 2005; Schwarzlose et al., 2005).

As far as the first objective was concerned, our data indicated that the processing of the own-face involved specific activation of the right inferior frontal gyrus and of the right insula in comparison with the processing of another highly familiar person's face. This implication of the right inferior frontal gyrus is consistent with previous studies reporting that this region is involved in the distinction between self and others (Uddin et al., 2005a) or in the attentive processing of the own-face (Sugiura et al., 2000). The implication of the right (Kircher et al., 2000, 2001) and the left insula (Sugiura et al., 2000) in visual self-face recognition has also been reported earlier. This structure was also attributed a role in the sustained attention to the representation of the own-face (Sugiura et al., 2000). These results are consistent with a right hemispheric dominance model of self-recognition and self-awareness (Keenan et al., 2000c, 2003b; Platek et al., 2004a, 2006). We did not find any implication of the left fusiform although it has occasionally been previously reported. However, studies that reported activation in this region compared the self-face with an unfamiliar face (Kircher et al., 2000, 2001; Sugiura et al., 2005). Hence results of these studies could reflect a mere familiarity effect instead of a genuine self-effect. The processing of the colleague's face, compared with that of the self-face, showed differential activation in the superior temporal gyrus in both hemispheres and in the left precuneus. Activation was more reduced for the selfface than for the other familiar face in these regions. This result is consistent with several previous studies having reported that the activation is actually more reduced in the temporoparietal junction (Sugiura et al., 2005), in the left superior temporal gyrus and precuneus (Uddin et al., 2005a) for the self-face than for another familiar face. Sugiura and colleagues argued that these regions contain the representation of people's names and explained the greater deactivation in these regions when perceiving the self-face by covert naming that is more likely to occur when seeing familiar faces than when seeing one's face.

As for the recognition of the familiar bodies, we tentatively reported activation in the right cingulate gyrus and in a large frontal area on the right side when perceiving the self-body. This is quite consistent with results that we reported here above and with previous findings related to self-face processing (Keenan et al., 2003b, Kircher et al., 2000; Platek et al., 2004a, 2006; Sugiura et al., 2000; Uddin et al., 2005a). It is also interesting that, as it was the case for faces, the insula was implicated in self-body perception but on the left side. In order to prevent an identification based on other cues than the body shape itself, our participants' garment was standardized and it could be that the lack of strong activation for this contrast was due to the fact that person identification is less easy or less reliable from bodies than from faces leading to less sensible activation. Consistently with this hypothesis, reaction times indicated that participants were faster for faces than for bodies. However, the fact that our participants were all females may limit the generalization of our results. Indeed, a recent study by Kurosaki et al. (2006) showed that some differences can be found between men and women when they are discriminating altered from intact versions of their own body. They showed that, for women, the confrontation to an altered version of the own body elicited activity in prefrontal and limbic areas (a parallel can be done with present observations) and for men, it rather elicited activity in the right occipital cortex. For the authors, this suggests that women would perceive distorted images of themselves by complex cognitivo-emotional processing whereas for men a more visuo-spatial processing is involved. However, contrary to us, these authors were interested in the processing of distorted images of oneself and they did not report data related to intact items. This makes hazardous any comparison between their study and the present one. Nevertheless, their findings related to gender differences should encourage further studies about familiar and self-body processing including males as well as females.

The last aim of this study was to investigate whether cerebral regions are activated by self-processing independently of the stimulus domain, i.e., regardless of whether a face or a body was processed. A comparison of the processing of self-related pictures with the processing of pictures related to another highly familiar person revealed an implication of the right anterior insula and of the right dorsal anterior cingulate. This implication of the anterior cingulate is in line with findings of a recent study by Platek et al. (2005). They showed that this region is at play during processing of self facial resemblance. Since this region is also implicated during the processing of face familiarity or self-referent information, they suggested that this region might be generally 
involved when making decisions about self-referential information. Our findings are also consistent with Northoff and Bermpohl (2004)'s thesis that the cingulate gyrus could play a role in abstract self-processing, that is, independent of the stimulus domain or of the sensorial modality. The activity found in the right anterior insula is also in line with previous studies investigating different aspects of self-processing and indicating that this structure is implicated in self-agency (Farrer and Frith, 2002), autobiographical episodic memory retrieval (Fink et al., 1996) and self-face recognition or self-descriptive judgments (Kircher et al., 2000, 2001). The specific role of this structure remains to be investigated but present results as well as those of previous studies suggest that, like the anterior cingulate, the right anterior insula could play a general role in making decisions about oneself. The right hemispheric implication is also in agreement with patient studies showing that the condition of persons suffering from an alteration of the sense of self is principally underlain by brain damages localized in the right frontal lobe (for a review see Feinberg and Keenan, 2005).

A secondary aim of this study was to check which regions are implicated during face or during body processing. Comparisons of body shape or face processing with seeing a scrambled image indicated that faces and bodies seemed to recruit close but segregated areas of the occipital cortex (see Fig. 1). This is in agreement with previous studies that identified distinct regions of the extrastriate cortex that are specifically devoted to faces (ventral occipital face area, OFA, Puce et al., 1996; Peelen and Downing, 2005) and bodies (extrastriate body area, EBA, Downing et al., 2001; Peelen and Downing, 2005). Visual analysis of Fig. 1 also indicated a common implication of the right fusiform gyrus for faces and bodies. This is also consistent with recent studies (Peelen and Downing, 2005; Schwarzlose et al., 2005) which found such overlapping although distinct regions of the fusiform gyrus were associated with presentation of faces and bodies. In addition, as it has already been shown previously (Spiridon et al., 2006), although left and right hemispheres showed a similar pattern of activation with faces and bodies (FFA and EBA), the implicated areas seemed less extended on the left than on the right. However, these results have to be taken cautiously because the two stimulus domains were presented in two different sessions which certainly gave rise to a poor sensitivity.

From these results, it appears that after a partly segregated structural processing of the shape of faces and bodies in posterior areas, the distinction between self and others might be processed in more anterior regions. Selfrelated stimuli were specifically processed mainly in the right frontal gyrus and in the anterior insula compared with stimuli depicting another highly familiar person. In agreement with previous studies (for a review, see Northoff and Bermpohl, 2004), we found that specific areas in the right anterior insula and in the right dorsal cingulate gyrus are also devoted to integrative self-processing regardless of the stimulus domain.

Thus, our results suggest that posterior and anterior regions play different roles in person identification. Posterior regions (i.e., occipito-temporal cortex) seem to be involved, at a first level of processing, in the distinction of different aspects of persons (i.e., a rough classification as face or body). Indeed, our results indicated that these regions were differently recruited to process different body parts (i.e., the face alone vs. the headless body). The fusiform gyrus could then perform a more detailed structural encoding of stimuli (i.e., here the different body parts) and give a first assessment of the seen person's familiarity (see Rossion et al., 2003) before further processing. That could explain why we did not find activity in this region when we compared the processing of two highly familiar persons (and corollary why other studies comparing self-face recognition with unfamiliar face recognition did so). Regarding person identification from faces, the present hypothesis represents an intermediate view (see also Grill-Spector et al., 2004) between the hypothesis that FFA simply allows to classify a stimulus as a face (detection hypothesis, see Kanwisher et al., 1997), and the other hypothesis that this area is involved in individual identification of faces (see Gauthier et al., 2000; Haxby et al., 2000). In addition, our results are in agreement with studies that show that the FFA is involved in face processing but also during identification/sub-categorization of different classes of objects by experts (Xu, 2005; for reviews, see Tarr and Cheng, 2003; Tarr and Gauthier, 2000). Indeed, the fusiform gyrus was recruited during face as well as body processing, i.e., two different classes of "objects" that humans process with a relative expertise. At a later level of processing, anterior regions (i.e., mainly the frontal and superior temporal cortices, the anterior cingulate cortex and the anterior insula) may serve to distinguish different persons from each other and to access more abstract information about identity of familiar individuals (for instance people's names and semantic information about these persons). More specifically, the anterior insula and the anterior cingulate cortex would permit to distinguish oneself from others and give rise to an abstract representation of oneself that could possibly participate in maintaining a sense of self.

To conclude, this study showed that specific cortical regions, mainly the right frontal cortex and the insula, are implicated in visual self-recognition compared with visual processing of highly familiar and personally known persons. These results support the view (Keenan et al., 2000c, 2001; Platek et al., 2004a) that the right frontal cortex is preferentially recruited during self-recognition. In addition, our data indicated, in agreement with the findings of several new recent studies (Downing et al., 2001, 2006; Peelen and Downing, 2005; Schwarzlose et al., 2005), that the occipito-temporal cortex is more or less recruited depending on whether a face or a body is 
perceived. Finally, our study indicated that posterior regions would be involved in an increasingly detailed structural representation of different aspects of a person, whereas anterior regions within the right hemisphere (i.e., the anterior insula and the dorsal anterior cingulate) would be implicated in distinguishing between the self and others and in a more abstract representation of the self.

\section{EXPERIMENTAL PROCEDURE}

\section{1. Participants}

Twenty right-handed female students from the University of Liege aged between 18 and 27 years $(M=22.1, S D$ $=2.3$ ) participated. Participants were recruited by pairs so that each participant served as a colleague for another participant. Each participant had known her colleague for between 2 and 6.5 years $(\mathrm{M}=4$ years, $\mathrm{SD}=1.3)$. They had no history of psychological or neurological disorders and had a normal or corrected-to-normal (with contact lens) vision. The study was approved by the Ethics Committee of the Faculty of Medicine and of the Faculty of Psychology of the University of Liege, and was performed in accordance with the ethical standards described in the Declaration of Helsinki (1964). All participants gave their informed consent prior to their inclusion in the study.

\section{2. Materials}

Each participant's face was photographed, in a frontal position, and with a neutral expression. The pictures were then cropped and resized to be 450 pixels wide $x 600$ pixels high $\left(10^{\circ}\right.$ x $13.4^{\circ}$ at a viewing distance of $\left.90 \mathrm{~cm}\right)$ with the image manipulation software Gimp 2. We made two alterations of the face of each participant: the eyes were moved 16 pixels $\left(0.35^{\circ}\right)$ inwards or outwards (see Fig. 5). These alterations were chosen to be plausible but easily detectable (Brédart and Devue, 2006). We also took a picture of each participant's body wearing the same white T-shirt and a pair of blue jeans. Shoes were digitally re-colored in black. These pictures were cropped and resized to be 350 pixels wide $\times 600$ pixels high $\left(7.8^{\circ}\right.$ x $\left.13.4^{\circ}\right)$. The face was hidden by a $100 \times 100$ pixels black square and the background wall was light grey. We also made two alterations on the body of each participant (using Morph Man 2000): the waist-to-hip ratio (WHR) was decreased or increased of $10 \%$ respectively by enlarging or reducing the width of the hips (see Fig. 6). These alterations were chosen to fit face alterations (i.e., affecting the horizontal dimension of a relevant part of the stimulus and giving rise to easily detectable but plausible novel configuration). Such alterations were thus performed on female bodies exclusively. Indeed, men's WHR is typically between 0.85 and 0.95 (Singh, 1995) and increasing this ratio would have produced stimuli showing grotesque body shapes with a WHR higher than 1. Each participant was presented with the mirror-reversed versions of her 6 pictures (one intact and two altered faces, one intact and two altered bodies) and the normal-oriented versions of the 6 pictures of her colleague. For each pair, the luminance of the 12 resulting pictures was equated with Matlab 6 to prevent any low-level differences among these pictures. In addition, the pictures of the intact face and body of each participant were scrambled (with Matlab 6, see Figs. 5d and $6 \mathrm{~d})$.

Fig. 5 - Sample of face stimuli from one participant. From the original picture (b) the eyes were moved inwards to decrease the interocular distance of 16 pixels $(a)$ or outwards to increase the interocular distance of 16 pixels (c). For one of the two control sessions, the intact face was scrambled (d).

a. Interocular distance $\mathbf{- 1 6}$ pixels

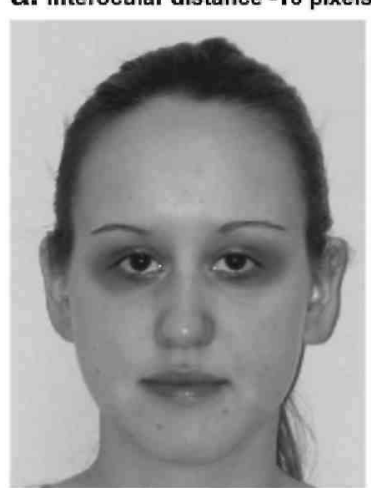

b. Intact face

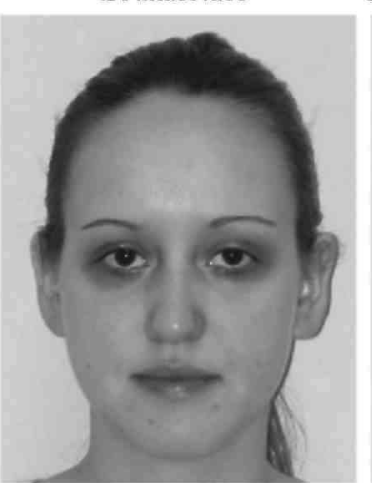

C. Interocular distance +16 pixels

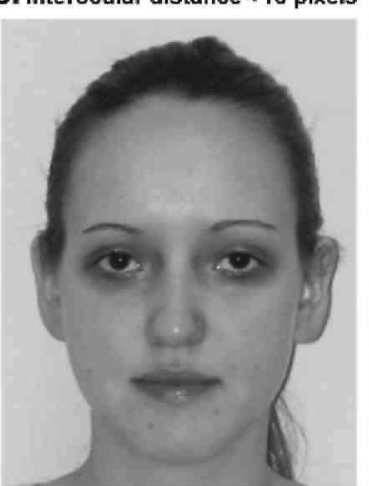

d. Scrambled intact face

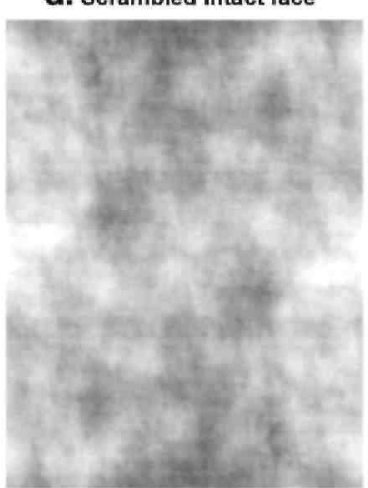


Fig. 6 - Sample of body stimuli from one participant. From the original picture $(b)$ the hips were made thinner to increase the WHR of 10\% (a) or wider to decrease the WHR of 10\% (c). For one of the two control sessions, the intact body was scrambled $(d)$.

a. Waist-hip ratio $+10 \%$

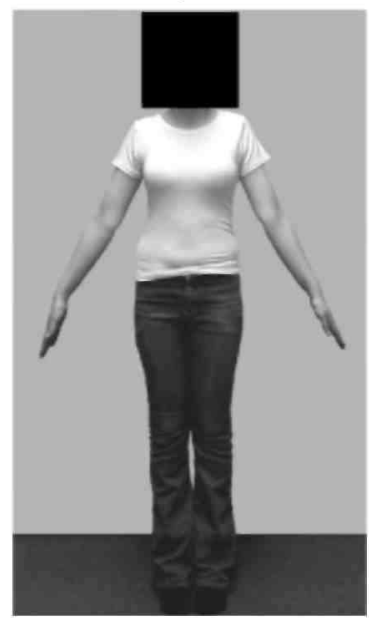

b. Intact body

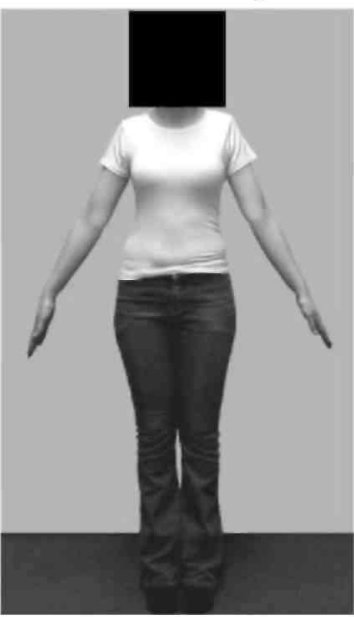

C. Waist-hip ratio $-10 \%$

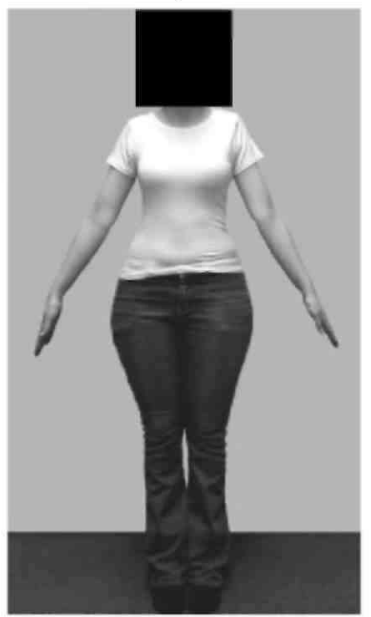

\section{d. Scrambled intact body}

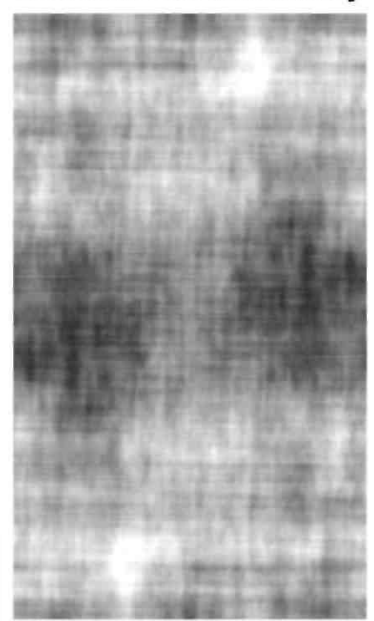

\section{3. Procedure}

Before being installed in the scanner, participants were presented 12 pictures: 3 pictures of their own face (the original one and the two altered versions), 3 pictures of their colleague's face, 3 pictures of their own body and 3 pictures of their colleague's body. The experimenter indicated whether each stimulus was intact or altered. The 'Stimulus appearance' factor hereafter refers to the intact vs. altered aspect of the stimulus while the 'Stimulus domain' factor defines whether a face or a body was presented. A pre-training (16 trials with faces and 16 trials with bodies) was administrated to illustrate the task to be performed in the scanner. The participants were then installed in the scanner. They hold a small keyboard in their right hand and stimuli were displayed on a black screen positioned at the back of the scanner, which the participant could comfortably see through a mirror fixed on the standard head coil.

We used an event-related paradigm within four different sessions. The two first sessions were the experimental sessions. Each one consisted of the presentation of the intact and altered versions of the pictures of the two members of the pair. In one of them, only faces were presented, and in the other, only bodies were presented (the presentation order of the two sessions being counterbalanced across participants). Within one session, each intact picture was presented 40 times and each altered picture was presented 20 times (for a total of 40, as there were two alterations per original picture). An experimental session was then composed of 160 trials presented at random. Null events consisted in random presentation of a white cross identical to the fixation cross (40 occurrences) during the session. The participants were instructed to press a key with their index finger if the picture was intact and another key with their middle finger if the picture was altered. Performing such an 'intactaltered' judgment implied that participants had first to identify the owner of the face/body to be able to give their response. They were allowed $2000 \mathrm{~ms}$ to respond. The picture stimulus disappeared immediately after pressing the response key (allowing some random variation between the successive presentation of two trials), followed by the presentation of a fixation cross for $1500 \mathrm{~ms}$. When they did not respond in the imparted $2000 \mathrm{~ms}$, a noresponse was recorded. Before each of the two scanning sessions started, participants were again presented with all the corresponding pictures and performed a training of 16 trials. In the two first sessions, we were interested in directly contrasting self-processing from the processing of another highly familiar person. So, control items taking into account low level perceptual processes (such as scrambled figures) were not used, these low level processes being similar regardless of the kind of items presented.

The last two sessions were the control ones. During each of these sessions, participants saw the intact picture (of their own face in one session, of their own body in the other one, the order being the same as that of the experimental sessions) 40 times and the scrambled version of this picture 40 times, in a random order. Each control session was thus composed of 80 trials. The pictures were presented for $2000 \mathrm{~ms}$ and were preceded by a red fixation cross for $750 \mathrm{~ms}$. The participants' task was to attend to this fixation cross and to report an infrequent color change (the cross was yellow in $12.5 \%$ of the cases) by pressing a key. This procedure was used to maintain the participants' attention while they passively viewed the stimuli of interest. Here, self-referential items were compared with scrambled items in order to suppress low-level perceptual processes that are not specific to face or body identification. 


\section{4. MRI acquisition}

Data were acquired on a 3 Tesla scanner (Siemens, Allegra, Erlangen, Germany) using a T2* sensitive gradient echo EPI sequence $\left(\mathrm{TR}=2130 \mathrm{~ms}\right.$, TE $=40 \mathrm{~ms}$, FA 90 , matrix size $64 \times 64 \times 32$, voxel size $\left.3.4 \times 3.4 \times 3.4 \mathrm{~mm}^{3}\right)$. Thirty-two 3-mm thick transverse slices (FOV $22 \times 22 \mathrm{~cm}^{2}$ ) were acquired, with a distance factor of $30 \%$, covering the whole brain. Structural images were obtained using a Tl-weighted 3D MP-RAGE sequence $(\mathrm{TR}=$ $1960 \mathrm{~ms}, \mathrm{TE}=4.4 \mathrm{~ms}$, FOV 23x23 $\mathrm{cm}^{2}$, matrix size 256x256x176, voxel size 0.9x0.9x0.9 mm). In each experimental session, between 177 and 292 functional volumes were obtained. In each control session, between 112 and 122 functional volumes were obtained. The first three volumes were discarded to account for Tl saturation. Head movement was minimized by restraining the participant's head using a vacuum cushion.

\section{5. $f M R I$ analyses}

Data were preprocessed and analyzed using SPM2 software (Wellcome Department of Imaging Neuroscience). Preprocessing included motion correction, spatial normalization in MNI space, and spatial smoothing using a Gaussian kernel of $8 \mathrm{~mm}$ full width at half maximum.

For each participant, brain responses were estimated at each voxel, using a general linear model. In each experimental session (face and body), events modeled transient responses to the two identities (self and colleague) and to the two stimulus appearances (intact or altered). Only brain responses for correct responses were entered in the matrix design.

In each control session (face and body), events modeled transient cerebral responses to the self identity for intact or scrambled items.

Delta functions representative of these trials types were convolved with the canonical hemodynamic response. The design matrix also included the realignment parameters to account for any residual movement-related effect. Two contrasts were performed in order to explore the main effect of the presented picture (intact vs. scrambled) in each of the two control sessions (face vs. body, contrasts 1 and 2). In experimental sessions, we were interested in cerebral areas involved in the processing of original self-related pictures in comparison with that of pictures depicting another highly familiar person. Seven linear contrasts were performed by using intact items only. These included two contrasts assessing the effect of the Stimulus domain (face vs. body): the first explored the effect of intact face processing in comparison with the processing of intact bodies (contrast 3 ), and the second contrast assessed the effect of intact body processing in comparison with intact face processing (contrast 4). The next four contrasts were carried out to compare (i) the effect of self-face processing relative to the processing of another highly familiar face (self minus other, contrast 5, and other minus self, contrast 6), and (ii) the effect of own body processing relative to the processing of another highly familiar body (self minus other, contrast 7, and other minus self, contrast 8). Finally, the effect of self-processing regardless of the Stimulus domain (face and body) in comparison with another familiar person was determined (contrast 9). No statistical inference was made at this level (fixed effects). Summary statistic images were thresholded at $p<0.9$ (uncorrected) and these images were further smoothed (6-mm FWHM Gaussian kernel). They were then entered in a second-level analysis, corresponding to a random effects model, in order to account for inter-subjects variance in each contrast of interest. One-sample $t$ tests assessed the significance of the effects. The resulting $\mathrm{SPM}\{\mathrm{T}\}$ maps were thresholded at $p<0.001$. Statistical inferences were performed at the voxel or cluster level at $p<0.05$ corrected for multiple comparisons (Bonferroni corrections) across the entire brain volume.

Alternatively, when previous studies led us to expect that changes in brain responses would occur in certain specified areas, a small volume correction (Worsley, 1996) was computed on a $20 \mathrm{~mm}$ maximum radius sphere around the average coordinates published for the corresponding relevant location. However, for contrasts about which there was no clear a priori hypothesis because of a lack of previous literature (this is particularly true for the visual recognition of familiar bodies), we tentatively reported activation at a threshold of $p<0.001$, uncorrected for multiple comparisons.

\section{6. A priori locations of interest}

The following a priori locations of interest were used for small volume corrections, based on published coordinates in the literature about self-face recognition and self-processing (judgment of adjectives/sentences as self-descriptive and judgment of actions as self generated). Regarding the general self-processing, independently of the Stimulus domain, these regions concerned mainly the medial/right prefrontal cortex, the right anterior cingulate, the bilateral insula and the bilateral precuneus. As for self-face recognition, the a priori locations of interest were chosen from literature using contrasts as comparable to ours as possible (own face minus other highly familiar face and conversely for the reversed contrast). These regions concerned primarily the right inferior frontal cortex and the left fusiform gyrus. Concerning the a priori locations of interest related to the processing of faces and bodies during the control session, they referred to studies comparing the processing of faces and bodies, respectively, to that of other kind of materials (i.e., tools, Peelen and Downing, experiment 1 , 
2005; scrambled controls, Peelen and Downing, experiment 2, 2005; letters or textures, Puce et al., 1996). All stereotactic coordinates refer to the MNI space. When a single coordinate refers to several studies, it corresponds to the centroid of all the coordinates reported in these studies. The a priori locations of interest were the following ones:

\section{6. 1. General effect of self-processing}

Right/medial prefrontal cortex [10, 50, 20; 10, 53, 5] (Fossati et al., 2003; Kelley et al., 2002), right anterior cingulate gyrus [6, 35, 6] (Craik et al., 1999), anterior insula [-36, -2, 2; 40, 8, 3] (Farrer and Frith, 2002), precuneus [-4, -56, 34; 6, -3, 36] (Kircher et al., 2000, 2002; Ruby and Decety, 2001), left inferior frontal gyrus [-39, 9, 16] (Craik et al., 1999; Kircher et al., 2002), left superior frontal gyrus [-11, 21, 59] (Ruby and Decety, 2001; Seger et al., 2004).

\section{6. 2. Effect of self-face recognition (self-face minus other familiar face processing)}

Right inferior frontal gyrus [45, 25, 14] (Platek et al., 2004a; Uddin et al., 2005a), left inferior frontal gyrus [-38, 25, 23] (Kircher et al., 2000), right superior frontal gyrus [26, 34, 34; 20, 16, 56] (Platek et al., 2006), right middle frontal gyrus [26, -12, 46] (Platek et al., 2006), right medial frontal gyrus [6, 48, -12] (Platek et al., 2006), left middle temporal gyrus [-52, 4, -16; - 58, -6, -4] (Platek et al., 2006), left fusiform gyrus [-14, -83, -18] (Kircher et al., 2001), right inferior parietal lobule [50, -62, 40; 64, -24, 50; 42, -34, 38] (Platek et al., 2006; Uddin et al., 2005a).

\section{6. 3. Effect of other familiar face recognition (other familiar face minus self-face processing)}

Left anterior cingulate [-2, 24, -2] (Platek et al., 2006), left superior temporal gyrus [-54, -42, 12] (Uddin et al., 2005a), right middle temporal gyrus [70, -12, -16] (Uddin et al., 2005a), precuneus [0, -48, 38] (Uddin et al., 2005a).

\section{6. 4. General effect of face recognition (face minus other material processing)}

Right fusiform gyrus [39, -44, -22; 30, -55, -24; 31, -55, -24] (Peelen and Downing, 2005, Puce et al., 1996), left fusiform gyrus [-38, -60, -26; -39, -54, -28] (Puce et al., 1996), right ventral occipital cortex [39, -65, -25] (Peelen and Downing, 2005), right occipito-temporal/inferior occipital sulci [36, -67, -22; 38, -63, -23] (Puce et al., 1996), bilateral lateral neocortex [47, -66, -4; 43, -67, -8; -40, -76, -7] (Puce et al., 1996).

\section{6. 5. General effect of body recognition (body minus other material processing)}

Right fusiform gyrus [40, -43, -21; 41, -38, -25] (Peelen and Downing, 2005), bilateral inferior temporal sulcus $[45,-67,-1 ; 47,-64,3 ;-49,-77,6]$ (Peelen and Downing, 2005).

\section{Acknowledgments}

This research was supported by a grant from the Belgian Fonds de la Recherche Fondamentale Collective (Grant no. 8.4506.05-2.4539.05) to SB. CD is Scientific Research Worker, FC is Research Associate and PM is Research Director at the National Fund for Scientific Research (FNRS) of Belgium.

\section{References}

[1] Brady, N., Campbell, M., Flaherty, M., 2004. My left brain and me: a dissociation in the perception of self and others. Neuropsychologia $42,1156-1161$

[2] Brédart, S., Devue, C., 2006. The accuracy of memory for faces of personally known individuals. Perception 35, $101-106$.

[3] Craik, F.I.M., Moroz, T.M., Moscovitch, M., Stuss, D.T., Winocur, G., Tulving, E., Kapur, S., 1999. In search of the self: a positron emission tomography study. Psychol. Sci. 10, 26-34.

[4] Downing, P.E., Jiang, Y., Shuman, M., Kanwisher, N., 2001. A cortical area selective for visual processing of the human body. Science $293,2470-2473$.

[5] Downing, P.E., Chan, A.W.-Y., Peelen, M.V., Dodds, C.M., Kanwisher, N., 2006. Domain specificity in visual cortex. Cereb. Cortex 16, $1453-1461$

[6] Farrer, C., Frith, C.D., 2002. Experiencing oneself vs another person as being the cause of an action: the neural correlates of the experience of agency. NeuroImage 15, 596-603.

[7] Feinberg, T.E., Keenan, J.P., 2005. Where in the brain is the self? Conscious. Cogn. 14, 661-678.

[8] Fink, G.R., Markowitsch, H.J., Reinkemeier, M., Bruckbauer, T., Kessler, J., Heiss, W.-D., 1996. Cerebral representation of one's own past: neural networks involved in autobiographical memory. J. Neurosci. 16, 4275-4282.

[9] Fossati, P., Hevenor, S.J., Graham, S.J., Grady, C., Keightley, M.L., Craik, F., Mayberg, H., 2003. In search of the emotional Self: an fMRI study using positive and negative emotional words. Am. J. Psychiatry 160, 1938-1945. 
[10] Gallup, G.G., Anderson, J.R., Platek, S.M., 2003. Self-awareness, social intelligence and schizophrenia. In: Kircher, T., David, A. (Eds. ), The Self in Neuroscience and Psychiatry. Cambridge Univ. Press, Cambridge, UK, pp. 147-165.

[11] Gauthier, I., Tarr, M.J., Moylan, J., Skudlarski, P., Gore, J.C., Anderson, A.W., 2000. The fusiform "face area" is part of a network that processes faces at the individual level. J. Cogn. Neurosci. 13, 495-504.

[12] Gillihan, S.J., Farah, M.J., 2005. Is self special? A critical review of evidence from experimental psychology and cognitive neuroscience. Psychol. Bull. 131, 76-97.

[13] Glila, T., Dehaene-Lambertz, G., 2005. Structural encoding of body and face in human infants and adults. J. Cogn. Neurosci. 17, 13281340

[14] Grill-Spector, K., Knouf, N., Kanwisher, N., 2004. The fusiform face area subserves face perception, not generic within-category identification. Nat. Neurosci. 7, 555-562.

[15] Grill-Spector, K., Henson, R., Martin, A., 2006. Repetition and the brain: neural models of stimulus-specific effects. Trends Cogn. Sci. $10,14-23$.

[16] Haxby, J., Hoffman, E., Gobbini, M.I., 2000. The distributed human neural system for face perception. Trends Cogn. Sci. 4, $223-233$.

[17] Holeckova, I., Fischer, C., Giard, M.-H., Delpuech, C., Morlet, D., 2006. Brain responses to a subject's own name uttered by a familiar voice. Brain Res. 1082, 142-152.

[18] Kanwisher, N., McDermott, J., Chun, M.M., 1997. The fusiform face area: a module in human extrastriate cortex specialized for face perception. J. Neurosci. 17, 4302-4311.

[19] Keenan, J.P., McCutcheon, B., Freund, S., Gallup, G.G., Sanders, G., Pascual-Leone, A., 1999. Left hand advantage in a self-face recognition task. Neuropsychologia 37, 1421-1425.

[20] Keenan, J.P., Freund, S., Hamilton, R.H., Ganis, G., Pascual-Leone, A., 2000a. Hand response differences in a self-face identification task. Neuropsychologia 38, 1047-1053.

[21] Keenan, J.P., Ganis, G., Freund, S., Pascual-Leone, A., 2000b. Self-face identification is increased with left hand responses. Laterality 5, 259-268.

[22] Keenan, J.P., Wheeler, M., Gallup, G.G., Pascual-Leone, A., 2000c. Self-recognition and the right prefrontal cortex. Trends Cogn. Sci. $4,338-344$

[23] Keenan, J.P., Nelson, A., O'Connor, M., Pascual-Leone, A., 2001. Self-recognition and the right hemisphere. Nature 409, 305.

[24] Keenan, J.P., Gallup, G.G., Falk, D., 2003a. The Face in the Mirror: The Search for the Origins of Consciousness. HarperCollins/Ecco, New York.

[25] Keenan, J.P., Wheeler, M., Ewers, M., 2003b. The neural correlates of self-awareness and self-recognition. In: Kircher, T., David, A. (Eds. ), The Self in Neuroscience and Psychiatry. Cambridge Univ. Press, Cambridge, UK, pp. 166-179.

[26] Keenan, J.P., Wheeler, M., Platek, S.M., Lardi, G., Lassonde, M., 2003c. Self-face processing in a callosotomy patient. Eur. J. Neurosci. 18, 2391-2395.

[27] Kelley, W.M., Macrae, C.N., Wyland, C.L., Caglar, S., Inati, S., Heatherton, T.F., 2002. Finding the self? An event-related fMRI study. J. Cogn. Neurosci. 14, 785-794.

[28] Kircher, T.T.J., Senior, C., Phillips, M.L., Benson, P.J., Bullmore, E.T., Brammer, M., Simmons, A., Williams, S.C.R., Bartels, M. David, A.S., 2000. Towards a functional neuroanatomy of self processing: effects of faces and words. Cogn. Brain Res. 10, $133-144$.

[29] Kircher, T.T.J., Senior, C, Phillips, M.L., Rabe-Hesketh, S., Benson, P.J., Bullmore, E.T., Brammer, M., Simmons, A., Bartels, M., David, A.S., 2001. Recognizing one's own face. Cognition 78, B1-B15.

[30] Kircher, T.T.J., Brammer, M., Bullmore, E., Simmons, A., Bartels, M., David, A. S., 2002. The neural correlates of intentional and incidental self processing. Neuropsychologia 40, 683-692.

[31] Kurosaki, M., Shirao, N., Yamashita, H., Okamoto, Y., Yamawaki, S., 2006. Distorted images of one's own body activates the prefrontal cortex and limbic/paralimbic system in young women: a functional magnetic resonance imaging study. Biol. Psychiatry 59, $380-386$.

[32] Northoff, G., Bermpohl, F., 2004. Cortical midline structures and the self. Trends Cogn. Sci. 8, 102-107.

[33] Peelen, M.V., Downing, P.E., 2005. Selectivity for human body in the fusiform gyrus. J. Neurophysiol. 93, 603-608.

[34] Perrin, F., Garcia-Larrea, L., Mauquiere, F., Bastuji, H., 1999. A differential brain response to the subject's own name persists during sleep. Clin. Neurophysiol. 110, 2153-2164.

[35] Perrin, F., Maquet, P., Peigneux, P., Ruby, P., Degueldre, C., Balteau, E., Del Fiore, G., Moonen, G., Luxen, A., Laureys, S., 2005. Neural mechanisms involved in the detection of our first name: a combined ERPs and PET study. Neuropsychologia 43, 12-19.

[36] Platek, S.M., Gallup, G.G., 2002. Self-face recognition is affected by schizotypal personality traits. Schizophr. Res. 57, 81-85.

[37] Platek, S.M., Keenan, J.P., Gallup, G.G., Mohamed, F.B., 2004a. Where am I? The neurological correlates of self and other. Cogn. Brain Res. 19, 114-122.

[38] Platek, S.M., Thomson, J.W., Gallup, G.G., 2004b. Cross-modal self-recognition: the role of visual, auditory, and olfactory primes. Conscious. Cogn. 13, 197-210.

[39] Platek, S.M., Keenan, J.P., Mohamed, F.B., 2005. Sex differences in the neural correlates of child facial resemblance: an event-related fMRI study. NeuroImage 25, 1336-1344. 
[40] Platek, S.M., Loughead, J.W., Busch, S., Ruparel, K., Phend, N., Panyavin, I.S., Langleben, D.D., 2006. Neural substrates for functionally discriminating self-face from personally familiar faces. Hum. Brain Mapp. 27, 91-98.

[41] Puce, A., Allison, T., Asgari, M., Gore, J., McCarthy, G., 1996. Differential sensitivity of human visual cortex to faces, letterstrings, and textures: a functional magnetic resonance imaging study. J. Neurosci. 16, 5205-5215.

[42] Rossion, B., Schiltz, C., Crommelinck, M., 2003. The functionally defined right occipital and fusiform "face areas" discriminate novel from visually familiar faces. NeuroImage 19, 877-883.

[43] Ruby, P., Decety, J., 2001. Effect of subjective perspective taking during simulation of action: a PET investigation of agency. Nat. Neurosci. 4, 546-550

[44] Schwarzlose, R.F., Baker, C.I., Kanwisher, N., 2005. Separate face and body selectivity on the fusiform gyrus. J. Neurosci. 25, 1105511059.

[45] Seger, C.A., Stone, M., Keenan, J.P., 2004. Cortical activations during judgments about the self and an other person. Neuropsychologia $42,1168-1177$

[46] Singh, D., 1995. Female judgment of male attractiveness and desirability for relationships: role of waist-to-hip ratio andfinancial status. J. Pers. Soc. Psychol. 69, 1089-1101.

[47] Sperry, R.W., Zaidel, E., Zaidel, D., 1979. Self-recognition and social awareness in the disconnected minor hemisphere. Neuropsychologia 17, 153-166

[48] Spiridon, M., Fischl, B., Kanwisher, N., 2006. Location and spatial profile of category-specific regions in human extrastriate cortex. Hum. Brain Mapp. 27, 77-89.

[49] Sugiura, M., Kawashima, R., Nakamura, K., Okada, K., Kato, T., Nakamura, A., Hatano, K., Itoh, K., Kojima, S., Fukuda, H., 2000. Passive and active recognition of one's own face. NeuroImage 11, 36-48.

[50] Sugiura, M., Watanabe, J., Maeda, Y., Matsue, Y., Kukuda, H., Kawashima, R., 2005. Cortical mechanisms of visual self-recognition. NeuroImage 24, 143-149.

[51] Tarr, M.J., Cheng, Y.D., 2003. Learning to see faces and objects. Trends Cogn. Sci. 7, 23-30.

[52] Tarr, M.J., Gauthier, I., 2000. FFA: a flexible fusiform area for subordinate-level visual processing automatized by expertise. Nat. Neurosci. 3, 764-769.

[53] Théoret, H., Kobayashi, M., Merabet, L., Wagner, T., Tormos, J.M., Pascual-Leone, A., 2004. Modulation of right motor cortex excitability without awareness following presentation of masked self-images. Cogn. Brain Res. 20, 54-57.

[54] Turk, D.J., Heartherton, T.F., Kelley, W.M., Funnell, M.G., Gazzaniga, M.S., Macrae, C.N., 2002. Mike or me? Self-recognition in a split-brain patient. Nat. Neurosci. 5, 841-842.

[55] Uddin, L.Q., Kaplan, J.T., Molnar-Szakacs, I., Zaidel, E., Iacoboni, M., 2005a. Self-face recognition activates a frontoparietal "mirror" network in the right hemisphere: an event-related fMRI study. NeuroImage 25, 926-935.

[56] Uddin, L.Q., Rayman, J., Zaidel, E., 2005b. Spilt brain reveals separate but equal self-recognition in the two cerebral hemisphere. Conscious. Cogn. 14, 633-640.

[57] Uddin, L.Q., Molnar-Szakacs, I., Zaidel, E., Iacoboni, M., 2006. rTMS to the right inferior parietal lobule disrupts self-other discrimination. Soc. Cogn. Affect. Neurosci. 1, 65-71.

[58] Worsley, K.J., 1996. A unified statistical approach for determining significant signals in images of cerebral activation. Hum. Brain Mapp. 4, 58-73.

[59] Xu, Y., 2005. Revisiting the role of the fusiform face area in visual expertise. Cereb. Cortex 15, 1234-1242.

[60] Zhu, Y., Qi, J., Zhang, J., 2004. Self-face identification in Chinese students. Acta Psychol. Sin. 36, $442-447$. 